\title{
Dor dentária como motivo de absenteísmo em uma população de trabalhadores
}

\author{
Dental pain as the motive for absenteeism in a sample of workers
}

Maria Helena Monteiro de Barros Miotto ${ }^{1}$

Jean Carlos Bazoni Silotti ${ }^{2}$

Ludmilla Awad Barcellos ${ }^{3}$

${ }^{1}$ Programa de Pós-

Graduação em Saúde

Coletiva, Universidade

Federal do Espírito Santo.

Avenida Marechal Campos

1468, Maruípe. 29040-090

Vitoria ES.

mhmiotto@terra.com.br

${ }^{2}$ Prefeitura Municipal de

Venda Nova do Imigrante

(ES).

${ }^{3}$ Faculdade de Odontologia,

Universidade de Vila Velha

(ES).
Abstract The aim of this study was to analyze the prevalence of dental pain, absenteeism induced by dental pain, and the association with sociodemographic characteristics. The research had a cross-sectional design using a random sample of 170 municipal employees from the City of Venda Nova do Imigrante, Espírito Santo state, Brazil, selected from a universe of 545 individuals. Data was collected using a 27-item questionnaire applied by a municipal worker. The comparison of dental pain and prevalence of absenteeism with socio-demographic and functional characteristics was verified using Fisher's Exact test. The research project was approved by an Ethics Research Committee. The prevalence of dental pain among the employees was $43 \%$ and half of these felt dental pains at work. Absenteeism related to dental pain was $23.4 \%$. Workers with lower instruction level were twice as likely to have dental pain $(O R=2,144, I C 95 \%=1,141 ; 4,030)$. Male subjects $(O R=5,714, I C 95 \%=1,773 ; 18,416)$, less years of education $(O R=4,875$, IC95\% = $1,405 ; 16,909)$ and less family income $(O R=3,613$, IC95\% $=0,931 ; 14,021)$ were related with higher absenteeism caused by dental pain. High dental pain frequency revealed in this study indicates that health promotion policies are needed to improve the quality of life of workers.

Key words Dental pain, Absenteeism, Occupational health, Public health
Resumo O objetivo foi analisar a prevalência da dor dentária e a ocorrência de absenteísmo provocado por dor de dente e as possiveis associações com características sociodemográficas. Um estudo analítico de corte transversal foi realizado em uma amostra aleatória de 170 sujeitos de um universo de 545 funcionários da Prefeitura Municipal de Venda Nova do Imigrante (ES). Para a coleta de dados foi utilizado um roteiro de 27 itens aplicado por uma servidora municipal. Para comparação dos percentuais entre dor dentária e absenteísmo com os fatores sociodemográficos foi utilizado o teste exato de Fisher. A prevalência de dor dentária entre os trabalhadores foi de $43 \%$ e a de absenteísmo por dor dentária foi de 23,4\%. Funcionários com grau de escolaridade até ensino médio incompleto relataram uma chance duas vezes maior de dor de dente $(O R=2,144$, IC95\% = 1,141;4,030). Trabalhadores do sexo masculino $(O R=5,714$, IC95\% = 1,773;18,416), de escolaridade até ensino médio incompleto $(O R=4,875$, IC95\% $=1,405 ; 16,909)$ e renda familiar até dois salários mínimos $(O R=3,613, I C 95 \%=$ 0,931;14,021) apresentaram maior prevalência de absenteísmo associada à dor de dente. A prevalência de dor encontrada demonstra a necessidade de políticas de promoção de saúde bucal para melhorar a qualidade de vida dos trabalhadores.

Palavras-chaves Dor dentária, Absenteísmo, Saúde do trabalhador, Saúde pública 


\section{Introdução}

Estudos epidemiológicos, nacionais e internacionais, demonstram que a ocorrência de dor é a razão principal pelas quais 75 a $80 \%$ das pessoas procuram os serviços de saúde; a dor crônica acomete entre 30 e $40 \%$ da população brasileira, representando a principal causa de absenteísmo, licenças médicas, aposentadorias por doença, indenizações trabalhistas e baixa produtividade no trabalho; a dor é uma das principais causas do sofrimento humano, gerando incapacidades, comprometimento da qualidade de vida e imensuráveis repercussões psicossociais e econômicas, constituindo-se, dessa forma, grave problema para a Saúde Pública ${ }^{1}$. As dores de dente fazem parte daquelas mais comuns que afetam a humanidade. Por causa do fracasso em manejar os determinantes sociais e materiais para incorporar saúde bucal dentro de promoção de saúde geral, milhões de pessoas sofrem dor de dente, têm pobre qualidade de vida e alta prevalência de dentes perdidos ${ }^{2}$.

Em decorrência de problemas odontológicos, as pessoas podem ter suas atividades diárias prejudicadas, tais como: diminuição das horas de sono, não realização de algumas atividades de lazer, restrições alimentares, desordens psicológicas relacionadas com a diminuição da autoestima e perda de dias e de aprendizagem na escola e/ou no trabalho. Estas implicações podem estar diretamente associadas à presença de dor ${ }^{3,4}$.

Dor dentária é capaz de produzir impacto na qualidade de vida. O que foi verificado por um estudo que encontrou prevalência diária entre adolescentes e suas famílias de 15,2\%. Uma das dimensões mais afetadas foi executar as atividades diárias domesticas ${ }^{5}$.

É importante avaliar os problemas bucais que podem atingir diretamente os trabalhadores, assim como estudar o impacto que eles podem ocasionar em sua qualidade de vida ${ }^{6}$.

O objetivo deste estudo foi analisar a prevalência da dor dentária nos últimos seis meses, a ocorrência de absenteísmo que provocou e possíveis associações com as características sociodemográficas nos funcionários da Prefeitura Municipal de Venda Nova do Imigrante (ES).

\section{Metodologia}

Este estudo populacional, observacional, analítico e transversal utilizou uma amostra representativa dos funcionários públicos municipais de
Venda Nova do Imigrante, município central do Estado do Espírito Santo, Brasil.

A partir de um universo de 545 funcionários públicos municipais, foi extraída uma amostra aleatória de 192 indivíduos utilizando uma listagem cedida pelo Setor Recursos Humanos da Prefeitura de Venda Nova do Imigrante (ES). O cálculo amostral utilizou como parâmetros uma prevalência esperada de $26 \%$, nível de confiança de $95 \%$ e margem de erro igual a $5 \%$.

O critério de inclusão adotado foi constar nome na lista de funcionários em plena atividade. Foram excluídos aqueles afastados por licença ou que estão exercendo cargo de confiança.

Foi realizado um projeto piloto com uma amostra de 20 funcionários públicos estaduais para possibilitar ajustes no instrumento de coleta de dados.

Um roteiro estruturado com 27 questões foi utilizado para a coleta de dados sociodemográficos, prevalência de dor dentária nos últimos seis meses e absenteísmo provocado por dor de origem dentária. Uma única funcionária pública, devidamente treinada, com o objetivo de manter a equivalência cultural e social com os participantes da pesquisa, realizou as entrevistas padronizadas no período de julho a setembro de 2007, após a obtenção do Termo de Consentimento Livre e Esclarecido.

A comparação dos percentuais entre dor dentária e absenteísmo por dor dentária com os fatores sociodemográficos e funcionais foi testada pelo teste exato de Fisher com significância de 5\%. Foi calculado o Odds Ratio (OR) e os respectivos intervalos de confiança.

A pesquisa foi conduzida dentro dos padrões exigidos pela Declaração de Helsinque e aprovada em julho de 2007, pelo Comitê de Ética em Pesquisa da Associação Brasileira de Odontologia, ES.

\section{Resultados}

Houve um predomínio do sexo feminino (73,5\%) entre os funcionários públicos municipais, com idade até 40 anos $(61,2 \%)$, escolaridade acima do ensino médio completo $(61,8 \%)$ e renda familiar até dois salários mínimos (64,1\%) (Tabela 1).

A grande maioria dos entrevistados $(67,65 \%)$ relatou alguma perda dentária; $11,3 \%$ indivíduos com perda total e 55,65\% com perda de um a quatro dentes. Em relação à necessidade percebida de prótese parcial removível (PPR), apenas $30,6 \%$ declararam precisar dela (Tabela 2). 
Tabela 1. Dados sociodemográficos dos funcionários públicos do município de Venda Nova do Imigrante (ES), 2007.

\begin{tabular}{lrr}
\hline \multicolumn{1}{c}{ Característica } & N & $\%$ \\
\hline Sexo & & \\
$\quad$ Masculino & 45 & 26,5 \\
$\quad$ Feminino & 125 & 73,5 \\
Faixa etária & 104 & 61,2 \\
$\quad$ 18 - 40 anos & 66 & 38,8 \\
$\quad 41$ anos ou mais & & \\
Nível de graduação & 65 & 38,2 \\
$\quad$ Até ensino médio incompleto & 105 & 61,8 \\
$\quad$ Acima de ensino médio completo & & \\
Renda familiar & 109 & 64,1 \\
$\quad$ Até dois salários mínimos & 61 & 35,9 \\
$\quad$ Acima de dois salários mínimos & $\mathbf{1 7 0}$ & $\mathbf{1 0 0 , 0}$ \\
Total &
\end{tabular}

Tabela 2. Situação da dentição e necessidade declarada de próteses dos funcionários públicos do município de Venda Nova do Imigrante (ES), 2007.

\begin{tabular}{lrc}
\hline \multicolumn{1}{c}{ Característica } & N & $\%$ \\
\hline Situação da dentição & & \\
$\quad$ Perda dentária & 55 & 32,35 \\
$\quad$ Ignorado ou não respondeu & 13 & 11,30 \\
$\quad$ Edentados & 64 & 55,65 \\
1 a 4 dentes perdidos & 24 & 20,88 \\
5 ou mais dentes perdidos & 14 & 12,17 \\
$\quad$ Ignorado o número dente perdido & & \\
Uso de PPR & 118 & 69,4 \\
$\quad$ Não precisa & 52 & 30,6 \\
$\quad$ Precisa & & \\
Uso de PT & 146 & 85,9 \\
$\quad$ Não precisa & 24 & 14,1 \\
Precisa & & \\
\end{tabular}

Foi verificada uma alta prevalência de dor dentária $(42,4 \%)$. Destes, 45 (62,5\%) visitaram o $\mathrm{CD}$, originando $23,6 \%$ de falta ao trabalho com $52,9 \%$ perdendo meio-turno de trabalho. A metade dos trabalhadores que sentiu dor de origem dentária compareceu ao trabalho (Tabela 3).

A comparação entre as variáveis sociodemográficas com a prevalência de dor dentária não foi significativa, apenas o grau de escolaridade foi estatisticamente associado. Trabalhadores com até ensino médio incompleto apresentaram maior prevalência de dor $(\mathrm{p}=0,013)$. Trabalha-
Tabela 3. Dor dentária e absenteísmo declarados pelos funcionários públicos do município de Venda Nova do Imigrante (ES), 2007.

\begin{tabular}{|c|c|c|}
\hline Característica & $\mathbf{N}$ & $\%$ \\
\hline \multicolumn{3}{|l|}{ Sentiu dor espontânea ou provocada } \\
\hline Sim & 72 & 42,4 \\
\hline Não & 98 & 57,6 \\
\hline \multicolumn{3}{|l|}{ Visita ao CD em caso de dor } \\
\hline Sim & 45 & 62,5 \\
\hline Não & 27 & 37,5 \\
\hline \multicolumn{3}{|l|}{ Tipo de serviço utilizado } \\
\hline Posto de saúde & 25 & 55,6 \\
\hline Particular & 20 & 44,4 \\
\hline \multicolumn{3}{|l|}{ Número de visitas ao $\mathrm{CD}$} \\
\hline Uma vez & 12 & 26,7 \\
\hline Duas vezes & 8 & 17,8 \\
\hline Três ou mais vezes & 25 & 55,4 \\
\hline \multicolumn{3}{|c|}{ Motivo da não utilização de serviço odontológico } \\
\hline Não sentiu necessidade & 9 & 33,3 \\
\hline Medo ou falta de dinheiro & 4 & 14,9 \\
\hline Outros motivos & 14 & 51,8 \\
\hline \multicolumn{3}{|c|}{ Precisou faltar ao trabalho por dor de dente } \\
\hline Sim & 17 & 23,6 \\
\hline Não & 55 & 76,4 \\
\hline \multicolumn{3}{|l|}{ Tempo de falta ao trabalho } \\
\hline Meio turno de trabalho & 9 & 52,9 \\
\hline Um a dois dias de trabalho & 8 & 37,1 \\
\hline \multicolumn{3}{|c|}{ Compareceu ao trabalho com dor de dente } \\
\hline Sim & 36 & 50,0 \\
\hline Não & 36 & 50,0 \\
\hline \multicolumn{3}{|c|}{ Faltou ao trabalho devido a alguma dor no corpo } \\
\hline Sim & 42 & 24,7 \\
\hline Não & 128 & 75,3 \\
\hline
\end{tabular}

dores com menor escolaridade relataram duas vezes mais chance de sentir dor de dente nos últimos seis meses $(\mathrm{OR}=2,144$ [IC 95\% 1,141; 4,30]) (Tabela 4).

A prevalência de absenteísmo foi maior em indivíduos do sexo masculino $(\mathrm{p}=0,004)$, com renda familiar até dois salários mínimos $(\mathrm{SM})$ $(\mathrm{p}=0,046)$ e com menor grau de escolaridade ( $\mathrm{p}$ $=0,009)$ (Tabela 5). Trabalhadores do sexo masculino relataram uma chance quase seis vezes maior de faltar ao trabalho por dor de dente $($ IC95\% $=1,773 ; 18,416)$.

Em relação à renda, a chance de um trabalhador faltar ao trabalho por dor de dente foi 3,6 vezes maior $($ IC95\% $=0,931 ; 14,021)$ para os que recebem até dois salários mínimos. Os trabalhadores com menor escolaridade relataram maior chance de absenteísmo (OR = 4,875 [IC95\% = $1,405 ; 16,909])$. 
Tabela 4. Dados sobre dor dentária e características sociodemográficas dos funcionários da Prefeitura Municipal de Venda Nova do Imigrante (ES), 2007.

\begin{tabular}{|c|c|c|c|c|c|c|}
\hline \multirow{2}{*}{ Característica } & \multicolumn{2}{|c|}{ Sentiu dor } & \multicolumn{2}{|c|}{ Não sentiu dor } & \multirow{2}{*}{ Sig. } & \multirow{2}{*}{ Odds } \\
\hline & $\mathbf{N}$ & $\%$ & $\mathbf{N}$ & $\%$ & & \\
\hline Sexo & & & & & 0,305 & 1,270 \\
\hline Masculino & 21 & 46,7 & 24 & 53,3 & & $(0,640 ; 2,520)$ \\
\hline Feminino & 51 & 40,8 & 74 & 59,2 & & \\
\hline Faixa etária & & & & & 0,208 & 1,360 \\
\hline Até 40 anos & 41 & 39,4 & 63 & 60,6 & & $(0,729 ; 2,538)$ \\
\hline 41 anos ou mais & 31 & 47,0 & 35 & 53,0 & & \\
\hline Renda familiar & & & & & 0,414 & 1,129 \\
\hline Até 2 salários mínimos & 45 & 41,3 & 64 & 58,7 & & $(0,599 ; 2,127)$ \\
\hline Mais de 2 salários mínimos & 27 & 44,3 & 34 & 55,7 & & \\
\hline Grau de escolaridade & & & & & 0,013 & 2,144 \\
\hline Até ensino médio incompleto & 35 & 53,8 & 30 & 46,2 & & $(1,141 ; 4,030)$ \\
\hline Acima de ensino médio completo & 37 & 35,2 & 68 & 64,8 & & \\
\hline
\end{tabular}

Tabela 5. Dados sobre absenteísmo por causa de dor dentária dos funcionários da Prefeitura Municipal de Venda Nova do Imigrante (ES), 2007.

\begin{tabular}{|c|c|c|c|c|c|c|}
\hline \multirow[b]{2}{*}{ Característica } & \multicolumn{2}{|c|}{$\begin{array}{l}\text { Faltou } \\
\text { trabalho }\end{array}$} & \multicolumn{2}{|c|}{$\begin{array}{l}\text { Não faltou } \\
\text { trabalho }\end{array}$} & \multirow[b]{2}{*}{ Sig. } & \multirow[b]{2}{*}{ Odds } \\
\hline & $\mathbf{N}$ & $\%$ & $\mathbf{N}$ & $\%$ & & \\
\hline Sexo & & & & & 0,004 & 5,714 \\
\hline Masculino & 10 & 47,6 & 11 & 52,4 & & $(1,773 ; 18,416)$ \\
\hline Feminino & 7 & 13,7 & 44 & 86,3 & & \\
\hline Faixa etária & & & & & 0,457 & 1,236 \\
\hline Até 40 anos & 9 & 22,0 & 32 & 78,0 & & $(0,414 ; 3,690)$ \\
\hline 41 anos ou mais & 8 & 25,8 & 23 & 74,2 & & \\
\hline Renda familiar & & & & & 0,046 & 3,613 \\
\hline Até 2 salários mínimos & 14 & 31,1 & 31 & 68,9 & & $(0,931 ; 14,021)$ \\
\hline Mais de 2 salários mínimos & 3 & 11,1 & 24 & 88,9 & & \\
\hline Grau de escolaridade & & & & & 0,009 & 4,875 \\
\hline Até ensino médio incompleto & 13 & 37,1 & 22 & 62,9 & & $(1,405 ; 16,909)$ \\
\hline Acima de ensino médio completo & 4 & 10,8 & 33 & 89,2 & & \\
\hline
\end{tabular}

\section{Discussão}

A prevalência de dor dentária entre os participantes deste estudo, realizado no município de Venda Nova do Imigrante (ES), foi de 42,4\%, com resultados similares aos encontrados em Toronto, Canadá $(39,7 \%)^{7}$; em Johore Bahru $(43,6 \%)^{8}$; e na Austrália $(51,9 \%)^{9}$. Esses resultados são diferentes dos dados do Reino Unido $(28 \%)^{10}$. Os estudos realizados em várias partes do Brasil apresentam resultados com maiores variações. Em Recife, em uma amostra de adolescentes, 33,6\% declararam dor nos últimos seis meses $^{4}$; no Rio de Janeiro em uma amostra de puerperais com média de 24 anos de idade, foi registrada uma prevalência de dor dentária de $39,1 \%{ }^{11}$; em Florianópolis (SC), 33,7\% dos escolares de 12 e 13 anos de idade declararam dor dentária $^{12}$, já os jovens de 18 anos do sexo masculino relataram uma frequência de $18,7 \%{ }^{13}$; em Porto Alegre, em uma amostra de adultos, constatou-se $20,7 \%{ }^{14}$; e em Natal, 29,6\% ${ }^{15}$. Um dado que chama atenção foi a alta prevalência de dor orofacial $(66,1 \%)$ em trabalhadores do setor metalúrgico e mecânico em Xanxerê (SC) ${ }^{16}$. As comparações entre esses estudos têm limitações 
devido a diferenças na faixa etária, populações, amostras e variações no período considerado para avaliar a frequência da dor que variou de 15 dias a 12 meses nos diversos estudos citados.

Com relação ao sexo, renda e faixa etária, este estudo não encontrou diferenças estatisticamente significantes na prevalência de dor dentária. Análise sobre faixa etária em estudos que envolvem dor de dente e absenteísmo tem sido dificultada pelas diferenças relacionadas ao ponto de corte para a formação dos grupos. Com referência à escolaridade, funcionários que estudaram até o ensino médio incompleto tiveram maior prevalência no relato de dor dentária $(53,8 \%)$. Resultados semelhantes foram relatados em outros estudos ${ }^{17-19}$. É consenso que renda e escolaridade são duas variáveis que apresentam forte associação, fato que não aparece neste estudo, talvez porque maior escolaridade não tenha sido traduzida em renda, possivelmente pelo fato de o salário recebido pelos funcionários públicos apresentar uma grande similaridade. Neste estudo os dados relativos à renda utilizados para análise foram declarados pelos trabalhadores; não foi considerada a possibilidade do funcionário ter outra fonte de renda, podendo ser considerada uma limitação deste estudo.

O impacto produzido por dor de dente envolve desde perda de horas de sono, diminuição da atenção, queda na produtividade, aumento do risco de acidentes de trabalho até a ausência no local de trabalho. Neste estudo, constatou-se que a dor foi suficientemente importante para afastar do trabalho $17(10 \%)$ participantes da pesquisa nos últimos seis meses.

Dos 72 indivíduos (42\%) que relataram dor de dente nos últimos seis meses, 17 (23,6\%) faltaram ao trabalho. Em Curitiba, uma pesquisa verificou 23,09\% de absenteísmo por motivo odontológico em trabalhadores de uma agroindústria ${ }^{20}$. $\operatorname{Nardi}^{19}$ encontrou uma prevalência de absenteísmo por dor dentária de 16,2\%. Reisine ${ }^{21}$ constatou que 25\% dos entrevistados relataram um episódio de falta ao trabalho devido a visitas ao CD por problemas odontológicos, e Alexandre et al. ${ }^{22}$ verificaram que a prevalência da dor dentária que impediu a realização de tarefas habituais foi de 2,9\%, diferença essa explicada pelo fato de o período da dor declarada ter sido nas últimas duas semanas; em contrapartida, este estudo e o de $\mathrm{Nardi}^{19}$ avaliaram a frequência de dor e absenteísmo nos últimos seis meses. O estudo realizado em Xanxerê, Santa Catarina, encontrou uma ocorrência de absenteísmo de $9,3 \%$, embora a prevalência de dor orofacial $(66,1 \%)$ tenha sido muito alta ${ }^{16}$.
Com relação ao presenteismo, 50\% dos participantes deste estudo relataram trabalhar com dor dentária, podendo ter perda da concentração no trabalho ${ }^{9}$ e outras consequências, desde diminuição na produtividade ${ }^{8}$ até acidentes de trabalho ${ }^{23}$. Este estudo vai ao encontro dos achados de Tauchen ${ }^{20}$ em que a grande maioria dos participantes relatou que já trabalhou com dor dentária (59,48\%). Mais estudos envolvendo a associação entre comparecimento ao trabalho, dor de dente e acidentes de trabalho são necessários com o propósito de planejar serviços odontológicos que garantam acesso aos trabalhadores. O ideal seria o serviço odontológico funcionando dentro da própria empresa, melhorando a qualidade de vida dos trabalhadores.

Nesta pesquisa, constatou-se que $62 \%$ dos funcionários que apresentaram dor de dente procuraram por cirurgião-dentista (CD), utilização considerada alta, principalmente quando comparada com estudos internacionais. Locker e Grushka ${ }^{7}$ observaram que $44 \%$ procuraram serviço odontológico para alívio da dor dentária. A pesquisa de Jaafar et al. ${ }^{8}$ verificou que $26 \%$ das pessoas com dor procuraram CD para tratamento, $38,1 \%$ não fizeram nada esperando que a dor desaparecesse, o que sugere que dor dentária nem sempre é capaz de gerar demanda. Por sua vez, Alexandre et al. ${ }^{22}$ encontraram que 13,3\% daqueles que sentiram dor dentária relataram nunca ter ido ao CD, mesmo sendo funcionários públicos de uma universidade que possui Curso de Odontologia.

Este estudo encontrou que os participantes do sexo masculino (47\%) relataram maior prevalência de absenteísmo por dor dentária, resultado homogêneo a outros estudos ${ }^{19,24,25}$. Em pesquisa realizada em uma empresa agropecuária de Minas Gerais, 33\% dos trabalhadores declararam absenteísmo por causas odontológicas. A prevalência foi maior no sexo feminino ${ }^{26}$. Em outros estudos, as mulheres apresentaram maior probabilidade de absenteísmo do que os homens somente no aspecto de assiduidade às visitas odontológicas de interesse preventivo ${ }^{27,28}$.

Funcionários com renda familiar de até dois salários mínimos e com menor grau de escolaridade apresentaram maior relato de absenteísmo por dor dentária, corroborando outros estu$\operatorname{dos}^{19,21,27,29}$.

Dos participantes que procuraram atendimento odontológico por dor dentária, 55,6\% optaram pelo serviço público e $44,4 \%$ procuraram atendimento particular. Aqueles que necessitaram de três ou mais visitas e foram atendidos 
na unidade de saúde faltaram mais ao trabalho, possivelmente porque o horário de atendimento nas unidades de Saúde coincide com o horário de trabalho do serviço público municipal ou, ain$\mathrm{da}$, por questões envolvendo resolutividade. Mais estudos devem ser realizados para oferecer respostas envolvendo o processo de trabalho nas unidades de saúde. Ações para maior resolutividade devem ser direcionadas à atenção básica com o intuito de modificar a realidade observada neste estudo, onde indivíduos do setor público ainda apresentam um alto percentual de dor capaz de impactar a qualidade de vida.

\section{Considerações finais}

A prevalência de dor foi muito expressiva e associada à escolaridade dos trabalhadores. A dor de dente observada foi responsável e capaz de produzir absenteísmo na população observada. Os resultados encontrados refletem dificuldade no acesso ao serviço odontológico e uma necessidade urgente de medidas de promoção de saúde e assistenciais direcionadas à população trabalhadora, reduzindo o sofrimento experimentado pelo agravamento dos problemas bucais e a exposição aos riscos do absenteísmo, como redução salarial e até mesmo demissão. A prevalência de dor encontrada demonstra necessidade da implementação das ações já regulamentadas no Brasil. A criação de um terceiro turno de atendimento no serviço público e/ou a inserção do CD nas empresas são consideradas estratégias capazes de melhorar o acesso e a qualidade de vida dos trabalhadores.

\section{Colaboradores}

MHMB Miotto participou da concepção da pesquisa, na metodologia e na redação final do artigo. JCB Silotti participou da coleta de dados e da redação final do artigo. LA Barcellos participou da concepção da pesquisa, na metodologia e na redação final do artigo. 


\section{Referências}

1. Brasil. Portaria n. ${ }^{\circ}$ 19/GM, de 3 janeiro de 2002 . Diário Oficial da União 2002; 3 jan.

2. Sheiham A. Oral health, general health and quality of life. Bull World Health Organ [serial on the Internet] 2005 Sept [cited 2007 Mar 7]; 83(9). Available from: http://www.scielosp.org/scielo.php?script= sci_arttext\&pid=S0042-96862005000900004\&lng= en \&nrm=iso\&tlng=en

3. Shepherd M, Nadanovsky PE, Sheiham A. The prevalence and impact of dental pain in 8-year-old schoolchildren in harrow. Br Dent J 1999; 187(1):38-41.

4. Goes PSA, Watt RG, Hardy R, Sheiham A. The prevalence and severity of dental pain in 14-15 years old Brazilian schoolchildren. Community Dent Health 2007; 24(4):217-224.

5. Goes PSA, Watt RG, Hardy R, Sheiham A. Impacts of dental pain on daily activities of adolescents aged 14-15 years and their families. Acta Odontol Scand 2008; 66(1):7-12.

6. Miotto MHMB, Loureiro CA. Efeito das características sociodemográficas sobre a freqüência dos impactos dos problemas de saúde bucal na qualidade de vida. UFES Rev Odontol 2003; 5(2):6-13.

7. Locker D, Grushka M. The Impact dental and facial pain. J Dent Res 1987; 66(9):1414-1417.

8. Jaafar N, Rasak IA, Zain RB. The impact of oral and pain in an industrial population. Ann Acad Med 1989; 18(5):553-555.

9. Sanders AEE, Spencer AJ. Job characteristics and the subjective oral health of Australian workers. Aust N Z J Public Health 2004; 28(3):259-266.

10. Pau AKH, Croucher R, Marcenes WS. Dental pain: demographic correlates. Br Dent J 2007; 202(9):548549 .

11. Vieira BHOM. Prevalência e impacto da dor de dente em uma população de mulheres grávidas no Rio de Janeiro, Brasil. [tese]. Rio de Janeiro (RJ): Universidade do Estado do Rio de Janeiro; 2003.

12. Nomura LH, Bastos JLD, Peres MA. Prevalência de dor de dente e associação com cárie e condições socioeconômicas em escolares, sul do Brasil 2002. Braz Oral Res 2004; 18(2):134-140.

13. Bastos JLD, Nomura LHE, Peres MA. Dor de dente e sua relação com condições socioeconômicas e cárie dentária em adultos jovens do sexo masculino no Sul do Brasil. Cad Saude Publica 2005; 21(5):1416-1423.

14. Gomes AS. Avaliação dos impactos odontológicos no desempenho diário nos trabalhadores do departamento municipal de limpeza urbana de Porto Alegre [dissertação]. Porto Alegre (RS): Universidade Federal do Rio Grande do Sul; 2005.

15. Ferreira AAA, Piuvezam G, Werner CWA, Alves MSCF. A dor e a perda dentária: representações sociais do cuidado à saúde bucal. Cien Saude Colet 2006; 11(1):211-218.

16. Lacerda JT, Traebert J, Zambenedetti ML. Dor orofacial e absenteísmo em trabalhadores da indústria metalúrgica e mecânica. Saude Soc 2008; 17(4):182191.

17. Comunello MH. Dor dental e impacto nas atividades diárias: um estudo de prevalência em estudantes de 12 anos, Joaçaba e Herval d'Oeste, Brasil. [dissertação]. Joaçaba (SC): Universidade do Oeste de Santa Catarina; 2003.
18. Lacerda JT, Simionato EM, Peres KG, Peres MA, Traebert J, Marcenes W. Dor de origem dental como motivo de consulta odontológica em uma população adulta. Rev Saude Publica 2004; 38(3):453-458.

19. Nardi A. Dor orofacial, absenteísmo e qualidade de vida em trabalhadores do sul do Brasil. [dissertação]. Joaçaba (SC): Universidade do Oeste de Santa Catarina; 2005.

20. Tauchen ALO. A contribuição do trabalho no programa de saúde ocupacional: verificando as condições de saúde bucal de trabalhadores de uma agroindústria do sul do Brasil [dissertação]. São Paulo (SP): Universidade de São Paulo; 2006.

21. Reisine ST. Dental Disease and Work Loss. J Dent Res 1984; 63(9):1158-1161.

22. Alexandre GC, Nadanovsky P, Lopes CS, Faerstein E. Prevalência e fatores associados à ocorrência da dor de dente que impediu a realização de tarefas habituais em uma população de funcionários públicos no Rio de Janeiro, Brasil. Cad Saude Publica 2006; 22(5):1073-1078.

23. Gemeli TR. Aplicação e análise de metodologia investigatória de causalidade entre morbidez odontológica e acidentes de trabalho. In: Simpósio Nacional de Vigilância em Saúde do Trabalhador, Florianópolis. 2005 jul [acessado 2007 set 20]; 1. Disponível em: http//:www.saude.sc.gov.br.

24. Diacov N, Sá Lima JR. Absenteísmo odontológico. Rev Odontol UNESP 1988; 17(1/2):183-189.

25. Lima JRS. Absenteísmo por causa odontológica: análise comparativa entre funcionários da Prefeitura do Município de São José dos Campos e segurados do Instituto Nacional de Previdência Social: Inamps, 2001. Medcenter.com ${ }^{\circledR}$ Odontologia [periódico na internet]. 2001 [acessado 2007 maio 12]. Disponível em: http://www.odontologia.com.br http://www.odontologia.com.br/artigos.asp $? \mathrm{id}=22$ \&idesp $=12$ \&ler $=s$

26. Pereira MC, Oliveira MA, Araújo VE, Carvalho CM. Absenteísmo por causas odontológicas em uma empresa agropecuária da Região Sudeste do Estado de Minas Gerais. Rev Bras Pesq Saúde 2010; 12(1):14-18.

27. Gift H, Reisine ST, Larach DC. The social impact of dental problems and visits. Am J Public Health 1992; 82(12):1663-1668.

28. Lisbôa IC, Abegg C. Hábitos de higiene bucal e uso de serviços odontológicos por adolescentes e adultos do Município de Canoas, Estado do Rio Grande do Sul, Brasil. Epidemiol Serv Saúde 2006; 15(4):29-39.

29. Nardi A, Michel-Crosato E, Biazevic MGH, Crosato E, Pizzatto E, Queluz DP. Relationship between orofacial pain and absenteeism among workers in Southern Brazil. Braz J Oral Sci 2009; 8(1):50-54.

Artigo apresentado em 25/03/2011

Aprovado em 01/04/2011

Versão final apresentada em 04/07/2011 